\title{
A Rare BRAF Fusion in Advanced Rectal Cancer Treated with Anti-Epidermal Growth Factor Receptor Therapy
}

\author{
Hiroko Hasegawa $^{a}$ Masaaki Miyo $^{\mathrm{b}}$ Kiyoshi Moric Masayuki Mano $^{\mathrm{c}}$ \\ Hisashi Ishida ${ }^{a} \quad$ Eiji Mita $^{a}$ \\ aDepartment of Gastroenterology and Hepatology, National Hospital Organization, Osaka \\ National Hospital, Osaka, Japan; bepartment of Surgery, National Hospital Organization, \\ Osaka National Hospital, Osaka, Japan; 'Department of Pathology, National Hospital \\ Organization, Osaka National Hospital, Osaka, Japan
}

\section{Keywords}

Advanced rectal cancer - Comprehensive genomic profiling · BRAF fusion · Anti-epidermal growth factor receptor therapy

\begin{abstract}
Recently, v-raf murine sarcoma viral oncogene homologue $B(B R A F)$ fusions have been identified in multiple cancer types using comprehensive genomic profiling (CGP) assays. BRAF fusions are extremely rare, occurring in $<0.5 \%$ of patients with metastatic colorectal cancer (mCRC). Until now, there is no standard treatment for $\mathrm{mCRC}$ with $B R A F$ fusions. Here, we report a recurrent colorectal cancer case that harbored an EXOC4-BRAF fusion. A 40-year-old female patient with a 2-year history of type 2 diabetes was diagnosed with pathologically confirmed stage IV rectal adenocarcinoma with liver metastasis. She underwent R0 resection after neoadjuvant therapy; however, her disease recurred at multiple metastatic sites (lymph nodes, ovary, and peritoneal gland). A rectal cancer surgical specimen was submitted for CGP (Foundation One) to identify potential targets to develop treatment strategies. An EXOC4$B R A F$ fusion was identified, and she achieved partial response to FOLFOX + panitumumab which is a fully human antibody directed against epidermal growth factor receptor. No EXOC4$B R A F$ fusions in colorectal cancer cases have been reported to date. Further studies investigating molecular mechanisms and novel targeted therapy approaches are required.
\end{abstract}



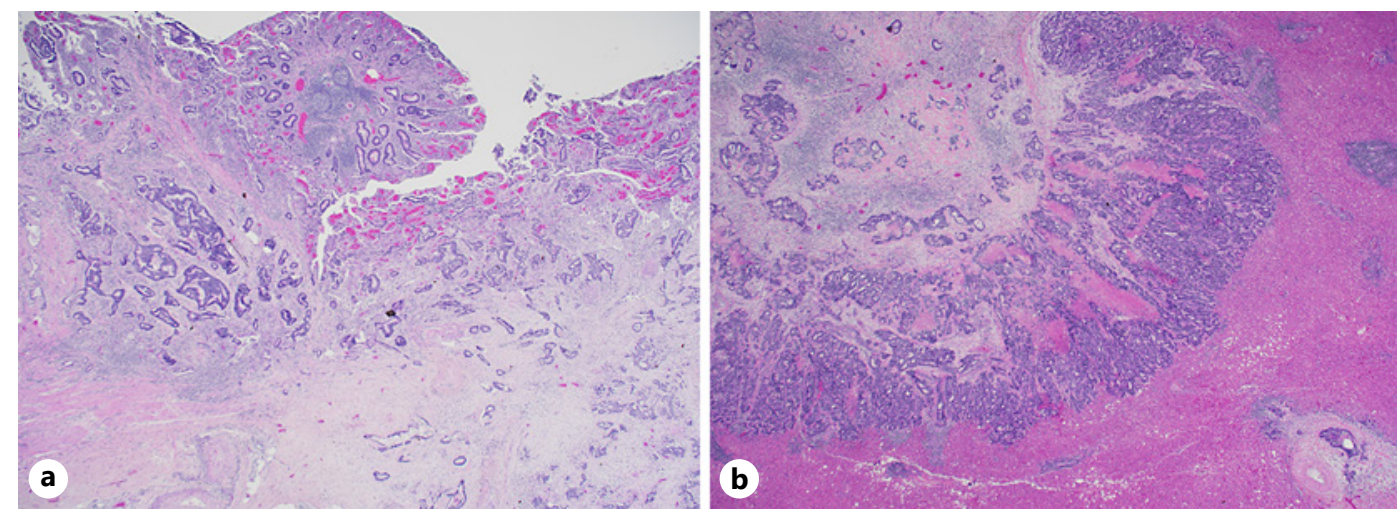

Fig. 1. Histopathological examination of the specimen of the resected rectal cancer $(\mathbf{a}, \times 30)$ and the liver metastasis $(\mathbf{b}, \times 30)$ with hematoxylin and eosin stain. Moderately differentiated adenocarcinoma with serosal invasion was diagnosed. Cancer cells are damaged without the destruction of the tumor structure which was consistent with grade 1a effects based on the Japanese Classification.

\section{Introduction}

v-raf murine sarcoma viral oncogene homologue $\mathrm{B}(B R A F)$ encodes a protein kinase that acts through the mitogen-activated protein kinase pathway (RAS/RAF/MEK/ERK pathway) and plays an important role in cell proliferation, differentiation, and survival [1]. BRAF alterations have become a major drug target and predictive biomarker in several malignancies [2]. The current understanding of BRAFV600E mutations originated in melanoma, where they are well-established biomarkers for cancer treatment and are observed in nearly $50 \%$ of cutaneous melanomas [3]. Moreover, in metastatic colorectal cancer (mCRC), BRAF mutation which has been identified in $8-10 \%$ of cancers is reportedly associated with resistance to anti-epidermal growth factor receptor (EGFR) therapy and a poor prognosis [4].

Recently, fusion genes which have been recognized as important cancer drivers thanks to advances in next-generation sequencing technologies have emerged as oncogenic drivers in several cancer types and represent potential targets for targeted therapy [5]. The BRAF gene is also reportedly involved in oncogenic gene fusions, with frequencies of $0.2-3 \%$ in some cancers [6]. Until now, $B R A F$ fusions in $\mathrm{mCRC}$ have been rarely reported; therefore, the impact of fusion partners on the oncogenic role of $B R A F$ and chemotherapy efficacy remains unknown.

Herein, we report a rare case of rectal cancer with lymph node and liver metastasis, with an EXOC4-BRAF fusion identified by clinical comprehensive genomic profiling (CGP) assay (Foundation One, Foundation Medicine, Cambridge, MA, USA). The patient was treated with anti-EGFR therapy and achieved a considerable clinical benefit.

\section{Case Report/Case Presentation}

A 40-year-old female patient with a 2-year history of type 2 diabetes was diagnosed with pathologically confirmed stage IV (T4aN1M1a) rectal adenocarcinoma (tub2) with liver (S7) metastasis. She received 6 cycles of capecitabine + oxaliplatin (XELOX) + bevacizumab (7.5 $\mathrm{mg} / \mathrm{kg}$ ) therapy as neoadjuvant treatment and underwent $\mathrm{R} 0$ resection both for liver metastasis (segmentectomy of S7) and primary rectal cancer in 2018. Pathological examinations revealed a poor response to neoadjuvant chemotherapy, which was consistent with grade 1a effects based on the Japanese Classification of Colorectal Carcinoma (Fig. 1). She then received 

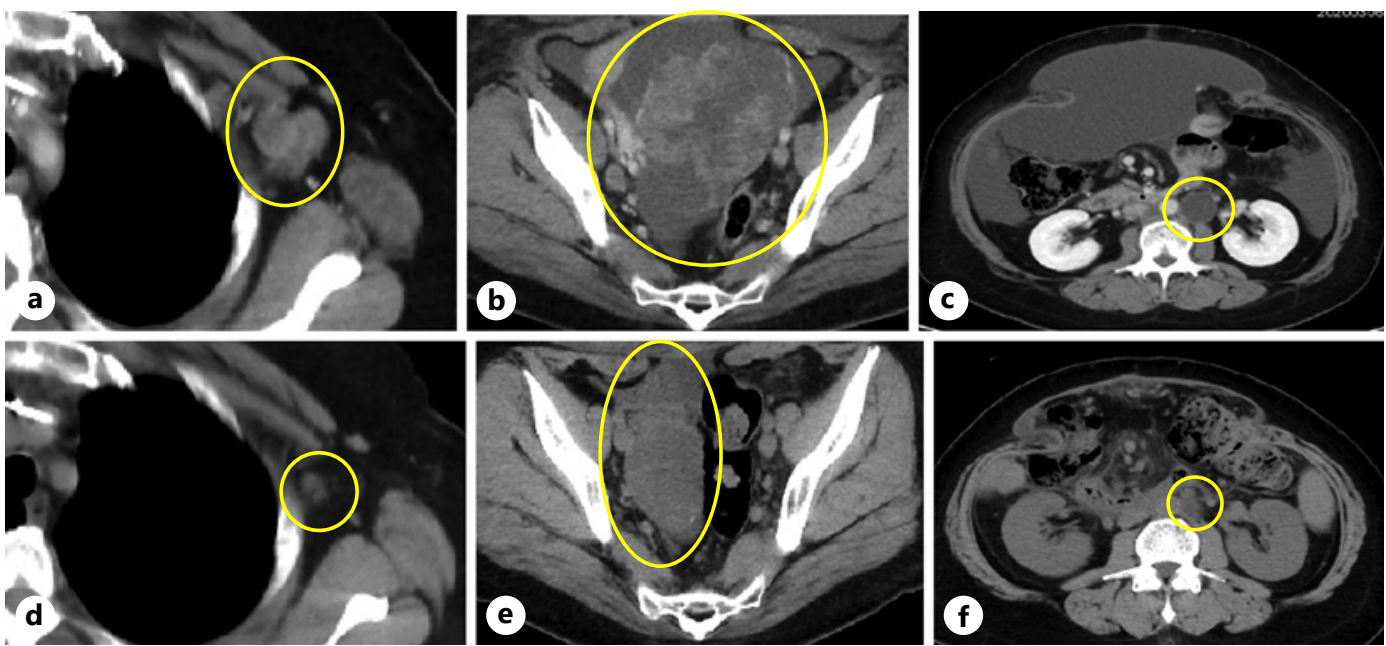

Fig. 2. Abdominal CT images before anti-EGFR combination therapy. Yellow circles indicate the metastatic sites of axillary lymph node (a), ovary (b), and para-aortic lymph node with ascites (c) Six months after the initiation of anti-EGFR combination therapy. $\mathbf{d - f}$ Decrease in size of the metastatic sites and disappearance of ascites (yellow circle). EGFR, epidermal growth factor receptor; CT, computed tomography.

XELOX as adjuvant chemotherapy for 6 months after surgery. However, 2 years after completion of adjuvant chemotherapy, the disease relapsed with multiple metastases in lymph nodes and ovary. Subsequently, she received FOLFIRI (irinotecan in combination with 5-fluorouracil (5-FU)/leucovorin) + bevacizumab therapy as palliative chemotherapy. A follow-up computed tomography scan after 3 FOLFIRI + bevacizumab cycles indicated that the size of lymph node metastases and ovarian metastasis had increased and moderate ascites extending from the pelvic to lower abdominal cavity due to the peritoneal metastases occurred. Because of a poor response to FOLFIRI + bevacizumab therapy, laboratory tests showed that carcinoembryonic antigen serum levels had increased from 40.0 to $52.0 \mathrm{ng} / \mathrm{mL}$ (normal range: $0-4.0 \mathrm{ng} / \mathrm{mL}$ ) and carbohydrate antigen 19-9 serum levels had increased from 129 to $192 \mathrm{U} / \mathrm{mL}$ (normal range: $0-40 \mathrm{U} / \mathrm{mL}$ ) when compared to before treatment commencement.

We also analyzed the mutational status of $R A S$ and $B R A F$ genes using polymerase chain reaction from surgical specimens of rectal cancer. No $R A S$ and $B R A F$ mutations were identified. A polymerase chain reaction-based assay for microsatellite instability did not reveal a high microsatellite instability profile. To explore potential targets for clinical trials or novel treatment strategies, rectal cancer surgical specimens were submitted for CGP to identify alterations in specific genes (Foundation One).

The CGP assay revealed mutations in $A B L 1$ (R712H), APC (R283), and TP53 (A138V), and a $B R A F$ fusion (EXOC4-BRAF) was also identified. The tumor mutation burden was 1.22 mut/ $\mathrm{Mb}$. At this point, no clinical trials were available for the patient to participate in; therefore, she continued with systematic chemotherapy. Given the presence of a primary left-sided colorectal tumor and no $R A S$ and $B R A F$ mutations, she received second-line palliative chemotherapy with FOLFOX-6 (oxaliplatin in combination with 5-FU/leucovorin) plus panitumumab which is a fully human antibody directed against EGFR. After six cycles, the patient achieved partial responses based on Response Evaluation Criteria in Solid Tumors version 1.1. Her ovarian metastasis, lymph nodes metastases, and massive ascites were decreased, and tumor markers were also decreased (carcinoembryonic antigen: $17.9 \mathrm{ng} / \mathrm{mL}$ and carbohydrate antigen 19-9: $22 \mathrm{U} / \mathrm{mL}$ ) (Fig. 2,3). Over 12 months, the patient continued on FOLFOX-6 plus panitumumab therapy, with the best response of partial response. 


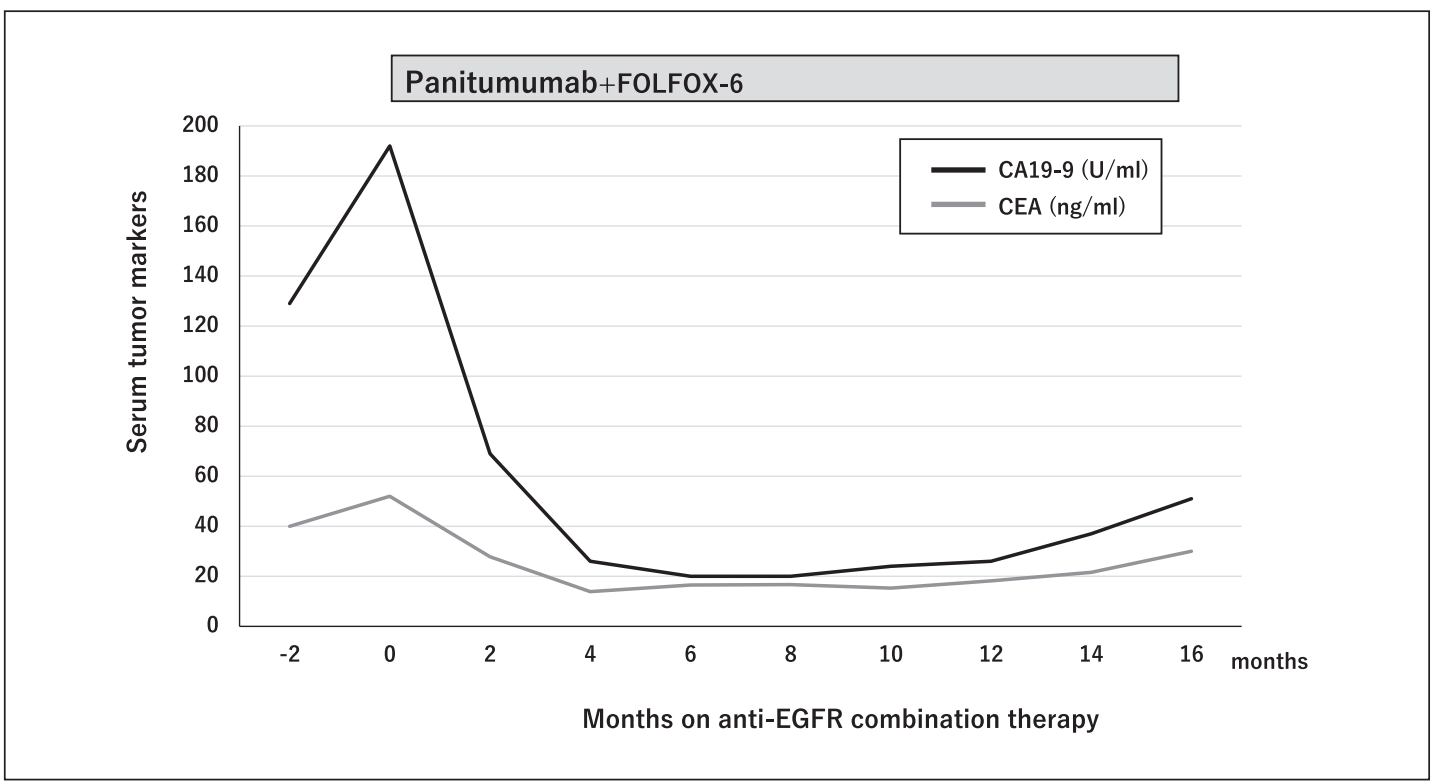

Fig. 3. Changes in serum level of tumor markers.

Table 1. Summary of cases with BRAF fusion in colorectal cancer

\begin{tabular}{ll}
\hline Author & $\begin{array}{l}\text { Types of BRAF fusion in } \\
\text { colorectal cancer }\end{array}$ \\
\hline Ross et al. [6] & MKRN1-BRAF \\
& TRIM24-BRAF \\
& $A G A P 3-B R A F$ \\
\hline Kloosterman et al. [10] & AGAP3-BRAF \\
& TRIM24-BRAF \\
& DLG1-BRAF \\
\hline Current case & EXOC4-BRAF \\
\hline
\end{tabular}

\section{Discussion}

Since the first $B R A F$ fusion case was identified in papillary thyroid carcinoma in 2005 [7], hundreds of tumors with the BRAF kinase domain fused to one or more partner genes (110 to date) have been identified in several cancer tumor types such as melanoma, lung, and prostate cancer $[6,8,9]$. In mCRC, $B R A F$ fusions are rarely identified, that is, $<0.5 \%$, according to a previous report [6]. Until now, some fusion partners of $B R A F$ in mCRC were reported $[6,10]$ (Table 1); however, the prognostic and biological implications of BRAF fusions are unclear.

In terms of anti-EGFR therapy responses, the presence of BRAFV600E mutations was previously identified as negative response predictors in $\mathrm{MCRC}$, based on sub-analysis data from phase III clinical trials [11]; however, it is currently unclear whether BRAF fusions in $\mathrm{mCRC}$ affect chemotherapeutic efficacy or prognosis.

To our knowledge, this is the first rare case report of responses to anti-EGFR therapy in mCRC where an EXOC4-BRAF fusion gene was identified. The exocyst complex component 4 (EXOC4) gene is located on the same chromosome as BRAF and is associated with insulinstimulated glucose transport related to type 2 diabetes [12]. 
According to a previous preclinical study [13], specific BRAF fusion genes (AGAP3$B R A F, D L G 1-B R A F$, and TRIM24-BRAF) were suggested to be relevant to the resistance of EGFR targeted therapy, same as BRAFV600E mutation; on the other hand, other novel $B R A F$ fusions were reported not to encode protein partners with obvious dimerization motifs associated with resistance of RAF inhibition [9]. It is unclear if these other novel fusions influenced the sensitivity to anti-EGFR therapy. In our case, the clinical features of this $B R A F$ fusion may be similar to non-V600E BRAF mutations in $\mathrm{mCRC}$, especially for class $3 B R A F$ mutations which were reportedly sensitive to anti-EGFR therapy owing to $R A S$ dependency [14].

For melanoma expressing $B R A F$ fusions, remarkable clinical response was achieved by MEK inhibition but not by BRAF inhibition [15]. For MEK inhibition, some $B R A F$ fusions such as PAPSS1-BRAF, TRIM24-BRAF, and FAM114A2-BRAF, ATG7-BRAF in melanoma, as well as ZC3HAV1-BRAF and FAM114A2-BRAF in thyroid cancer among various types of fusions which likely produce constitutively activated BRAF dimers independently of RAS were reported to be sensitive to MEK inhibitors [15]. To date, there is no data on the effective therapy for mCRC with $B R A F$ fusion, due to the rarity of this disease.

\section{Conclusion}

In conclusion, a comprehensive targeted therapy has not been established for mCRC patients harboring $B R A F$ fusions due to disease rarity. Further studies are required to investigate the clinical features of different $B R A F$ fusions, and similarly, additional research is needed to develop targeted therapeutic strategies for this fusion in MCRC.

At the time of this report, the patient was on the next palliative chemotherapy as salvage line therapy with stable disease 40 months after the first diagnosis. Compassionate use program based on JAPAN's system for MEK inhibitor is now being discussed.

\section{Acknowledgements}

The authors would like to thank the patient for providing consent to publish clinical information and data. We would like to thank ENAGO for the English language review.

\section{Statement of Ethics}

The case was approved by the Clinical Ethics Committee of the Osaka National Hospital. The written informed consent form was obtained from this patient to publish her case (including publication of images).

\section{Conflict of Interest Statement}

All the authors have no conflicts of interest to declare.

\section{Funding Sources}

The authors declare that there was no funding for this case report.

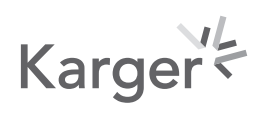




\section{Case Reports in Oncology}

\begin{tabular}{l|l}
\hline Case Rep Oncol 2021;14:938-943 \\
\hline DOI: 10.1159/000517007 & $\begin{array}{l}\text { @ 2021 The Author(s). Published by S. Karger AG, Basel } \\
\text { www.karger.com/cro }\end{array}$ \\
\hline
\end{tabular}

Hasegawa et al.: A Rare BRAF Fusion in Advanced Rectal Cancer

\section{Author Contributions}

H.H. involved in the case, collected the data of the case, and wrote the manuscript. M.M. and K.M. were responsible for patient diagnosis and treatment. E.M. and H.I. helped in writing and editing the case report. All the authors critically revised the manuscript, approved the final manuscript, and agreed to be accountable for all aspects of the work.

\section{References}

1 Davies H, Bignell GR, Cox C, Stephens P, Edkins S, Clegg S, et al. Mutations of the BRAF gene in human cancer. Nature. 2002 Jun 27;417(6892):949-54.

2 Turski ML, Vidwans SJ, Janku F, Garrido-Laguna I, Munoz J, Schwab R, et al. Genomically driven tumors and actionability across histologies: BRAF-mutant cancers as a paradigm. Mol Cancer Ther. 2016 Apr; 15(4):533-47.

3 Gonzalez D, Fearfield L, Nathan P, Tanière P, Wallace A, Brown E, et al. BRAF mutation testing algorithm for vemurafenib treatment in melanoma: recommendations from an expert panel. Br J Dermatol. 2013 Apr; 168(4):700-7.

4 Sanz-Garcia E, Argiles G, Elez E, Tabernero J. BRAF mutant colorectal cancer: prognosis, treatment, and new perspectives. Ann Oncol. 2017 Nov 1;28(11):2648-57.

5 Heydt C, Wölwer CB, Velazquez Camacho O, Wagener-Ryczek S, Pappesch R, Siemanowski J, et al. Detection of gene fusions using targeted next-generation sequencing: a comparative evaluation. BMC Med Genomics. 2021 Feb 27;14(1):62.

6 Ross JS, Wang K, Chmielecki J, Gay L, Johnson A, Chudnovsky J, et al. The distribution of BRAF gene fusions in solid tumors and response to targeted therapy. Int J Cancer. 2016 Feb 15;138(4):881-90.

7 Ciampi R, Knauf JA, Kerler R, Gandhi M, Zhu Z, Nikiforova MN, et al. Oncogenic AKAP9-BRAF fusion is a novel mechanism of MAPK pathway activation in thyroid cancer. J Clin Invest. 2005 Jan;115(1):94-101.

8 Zehir A, Benayed R, Shah RH, Syed A, Middha S, Kim HR, et al. Mutational landscape of metastatic cancer revealed from prospective clinical sequencing of 10,000 patients. Nat Med. 2017 Aug 4;23(8):1004.

9 Stransky N, Cerami E, Schalm S, Kim JL, Lengauer C. The landscape of kinase fusions in cancer. Nat Commun. 2014 Sep 10;5:4846.

10 Kloosterman WP, Coebergh van den Braak RRJ, Pieterse M, van Roosmalen MJ, Sieuwerts AM, Stangl C, et al. A systematic analysis of oncogenic gene fusions in primary colon cancer. Cancer Res. 2017 Jul 15;77(14): 3814-22.

11 Van Cutsem E, Köhne CH, Láng I, Folprecht G, Nowacki MP, Cascinu S, et al. Cetuximab plus irinotecan, fluorouracil, and leucovorin as first-line treatment for metastatic colorectal cancer: updated analysis of overall survival according to tumor KRAS and BRAF mutation status. J Clin Oncol. 2011 May 20;29(15):2011-9.

12 Laramie JM, Wilk JB, Williamson SL, Nagle MW, Latourelle JC, Tobin JE, et al. Polymorphisms near EXOC4 and LRGUK on chromosome 7q32 are associated with type 2 diabetes and fasting glucose; the NHLBI Family Heart Study. BMC Med Genet. 2008 May 22;9:46.

13 Stangl C, Post JB, van Roosmalen MJ, Hami N, Verlaan-Klink I, Vos HR, et al. Diverse BRAF gene fusions confer resistance to EGFR-targeted therapy via differential modulation of BRAF activity. Mol Cancer Res. 2020 Apr; 18(4):537-48.

14 Yaeger R, Kotani D, Mondaca S, Parikh AR, Bando H, Van Seventer EE, et al. Response to anti-EGFR therapy in patients with BRAF non-V600-mutant metastatic colorectal cancer. Clin Cancer Res. 2019 Dec 1;25(23): 7089-97.

15 Hutchinson KE, Lipson D, Stephens PJ, Otto G, Lehmann BD, Lyle PL, et al. BRAF fusions define a distinct molecular subset of melanomas with potential sensitivity to MEK inhibition. Clin Cancer Res. 2013 Dec 15; 19(24):6696-702. 